\title{
Valorization of Cocoa Husks: Pectin Recovery
}

\author{
Chiara Mollea and Fulvia Chiampo \\ Department of Applied Science and Technology, Politecnico di Torino, 10129 Torino, Italy \\ Correspondence should be addressed to Fulvia Chiampo; fulvia.chiampo@polito.it
}

Received 31 October 2018; Revised 5 February 2019; Accepted 21 February 2019; Published 1 April 2019

Academic Editor: Salam A. Ibrahim

Copyright (c) 2019 Chiara Mollea and Fulvia Chiampo. This is an open access article distributed under the Creative Commons Attribution License, which permits unrestricted use, distribution, and reproduction in any medium, provided the original work is properly cited.

\begin{abstract}
Food processing by-products are usually cheap and abundant and can be source of valuable molecules of great interest to various industries like the pharmaceutical or the food ones. In this frame, the husks of roasted cocoa beans, that are a by-product of the cocoa processing industry, can constitute a source of pectin. The recovery process has been already defined at laboratory scale with boiling acid extraction ( $\mathrm{pH}$ 2.5). This process is suitable to recover a quantity of pectin, expressed as anhydro-galacturonic acid (AGA), around $8 \mathrm{~g}$ AGA/100 g dry husks; this pectin is characterized by low degree of methylation (\%DM around 31) and acetylation degree lower than $2 \%$. In this paper the effects of some operative conditions on pectin quantity and quality were studied, in order to optimize the parameters that can make the process economically competitive: the in-excess quantities of solvents and operation time were reduced, without altering yield and pectin characteristics. In particular, the extract was concentrated by $13.3 \%$, the ethanol for pectin precipitation was reduced (ratio extract to ethanol equal to 1:4), and it was also demonstrated that a single washing with $40 \%$ ethanol is sufficient to obtain a purified product.
\end{abstract}

\section{Introduction}

In 2017 about 100 thousand tons of cocoa beans was imported in Italy, against a world production of 4.6 million tons and about the whole amount is ground [1]. Roasted cocoa beans are still covered by lignocellulosic husks which can be easily removed after roasting itself and before grinding. Considering the large amount of processed beans, the resulting volume of wasted husks is significant and can represent a disposal problem in those districts where there is a high concentration of companies working in the cocoa processing sector.

Reduction, recovery and recycling of organic residues are well known as three of the main environmental challenges for the food industry [2]. In this frame, food processing byproducts can be source of valuable molecules, of great interest for the pharmaceutical, cosmetic, and food industries $[3,4]$.

By this way, different uses of roasted cocoa husks can be found: looking at their chemical and physical characteristics, they are usually sent to combustion or exploited as fiber supplement for animal feeding or used to produce activated carbon $[5,6]$. As alternative, husks can be submitted to matter recovery operations becoming a source of additives or other high value products. In particular, at laboratory scale they have been already investigated as a potential source of pectin $[4,5,7]$.

Extraction conditions have a relevant influence on the chemical characteristics of the extracted pectin [8-10]. At industrial level, the extraction process is usually done by boiling acid, with hydrochloric acid as the most used (nitric, citric, and oxalic acid are common alternatives). After extraction, pectin precipitation is carried out with high concentration or absolute ethanol or isopropanol. At last, the precipitate is washed with high concentration (> 70\%) ethanol in single or multiple operations.

Previous laboratory runs to extract pectin from roasted cocoa husks gave yields between 6.0 and $8.4 \mathrm{~g}$ AGA/100 g dry husks, with the maximum yield obtained with boiling $\mathrm{HCl}$ solution ( $\mathrm{pH}$ 2.5) for one hour [7].

Since husks contain a low amount of pectin if compared with traditional sources (e.g., apple pomace and citrus peels), the whole recovery process must be competitive in order to make it attractive for the pectin commercialization. The process defined at laboratory scale utilizes quantities of solvents in excess and long process time. In view of an industrial process, these conditions are not sustainable since they would require high operating costs. For this reason, all 
the "excess" must be reduced, in terms of quantity of used chemicals and times optimization without changing the yield of extraction.

The operative conditions of the extraction step were widely studied, and scientific literature is rich of optimization data [7-13]. However, less attention was paid to the pectin precipitation and washing conditions, notwithstanding they are time and chemical consuming.

In this frame, the main aim of the present study was to optimize the process "economy"; in particular, the operations to recover and purify the extracted pectin, namely, alcohol precipitation and washing (APW), were investigated and their optimization to save time and chemicals amount was tried, even if they are the most commonly used techniques $[8-10,12]$.

\section{Materials and Methods}

2.1. Raw Materials and Chemicals. For this study husks of Venezuelan roasted cocoa beans were utilized; husks were ground in a grinding mill before pectin extraction.

All the chemicals and reagents were of analytical grade (Sigma Aldrich S.r.l. Milano, Italy). The kit for the enzymatic analysis of acetic acid (Cat. No. 10148261 035) was purchased from Boehringer Mannheim/R-BIOPHARM AG, Darmstadt, Germany.

2.2. Pectin Extraction and Recovery. A previous study by Mollea et al. [7] defined the procedure for pectin extraction and recovery by means of these steps:

(1) extraction with $\mathrm{HCl}$ solution at $\mathrm{pH} 2.5$ for 1 hour with ratio husks mass to $\mathrm{HCl}$ solution one equal to 1:25;

(2) precipitation with 7 volumes of boiling $99.8 \%$ ethanol for 20 minutes;

(3) one washing with 10 volumes of $63 \%$ ethanol.

The modifications studied in this paper are related to the operations no. 2 and no. 3 , and they were done in the order indicated in the following subchapters.

\subsection{Precipitation: Ethanol Quantity Reduction and Contact} Time Prolongation. At industrial level, the volume ratio of extract to alcohol may vary from 1:1 to $1: 5$ even if the ratio 1:3 allows a complete recovery [14].

The first analyzed change was the reduction of the ethanol quantity. As reference, the method by Mollea et al. [7] was considered: this method uses the ratio 1:7. Trials were performed using 4, 2 and 1 volumes of boiling $99.8 \%$ ethanol with respect to the extract volume.

The precipitation time was studied, too: the referential time (20 minutes) was increased threefold (1 hour) and sixfold ( 2 hours) for each extract-to-ethanol ratio. For the ratio 1:1 just the trial with the longest time ( 2 hours) was carried out. In all the runs, the temperature was $85^{\circ} \mathrm{C}$.

2.4. Precipitation: Extract Concentration. To minimize the volume of alcohol, the common industrial process usually concentrates the extract before precipitation. Thus, the extract was vacuum concentrated in order to remove partially the solvent by heating at $60^{\circ} \mathrm{C}$ and constant pressure. The extract was concentrated by $11.5 \%, 13.3 \%$, and $15.2 \%$ with respect to the initial volume; higher concentrations can cause pectin degradation [14].

For each concentrated extract the precipitation was done with ratio extract to $99.8 \%$ ethanol equal to $1: 4$ and precipitation time equal to 20 minutes.

2.5. Washing: Number of Operations. In order to control the purity of the final product, the runs with the three aforesaid concentrated extracts were also repeated modifying the washing operation. The pectin was washed twice with $63 \%$ ethanol.

The effect of the double washing was evaluated both in quantitative terms (as g AGA/100 g dry husks) to control that the higher washing volume did not cause pectin loss and in qualitative terms (as \%DM and acetylation degree) to check impacts on pectin quality.

2.6. Washing: Reduction of Ethanol Concentration. To reduce volumes of ethanol, the alcohol concentration in the washing solution was reduced. In subsequent experiments the effect of a lower ethanol concentration was studied in samples with extract concentrated by $13.3 \%$ of the starting volume (item 2.4): a single washing was used with the ethanol concentration reduced at 45,40 , and $35 \%$, respectively, instead of $63 \%$. Also in this case quantity and quality of pectin were controlled.

The set of experiments is summarized in Table 1.

2.7. Precipitation and Washing: Ethanol Reduction with $13.3 \%$ Concentrated Extract. Pectin recovery was carried out with the $13.3 \%$ concentrated extract and reducing the ratio extract to ethanol at 1:2 and 1:1 (20 minutes and 1 and 2 hours as precipitation times). The washing operation was single and with $40 \%$ ethanol.

2.8. Extraction Yield and Pectin Quality. Each experimental run was checked in terms of

(i) extraction yield, as anhydro-galacturonic acid content (g AGA/100 g dry husks) [15];

(ii) pectin quality, as \%DM [16] and acetyl ester content [7];

(iii) presence of polyphenols in solubilized pectin with the $\alpha$-naphthol method [17].

All analyses were carried out in triplicate.

\section{Results and Discussion}

3.1. Precipitation: Ethanol Quantity Reduction and Contact Time Prolongation. Figure 1 shows the results.

As regards the reduction of ethanol, getting from the ratio $1: 7$ to $1: 4$, the quantity of recovered pectin is slightly lower compared to the reference (1:7). When the ethanol quantity is additionally decreased (ratio 1:2), there is another limited 
TABLE 1: Set of experiments done changing: number of washings, ethanol concentration, and extract concentration with respect to the initial volume.

\begin{tabular}{|c|c|c|c|c|c|}
\hline \multirow[b]{2}{*}{ Number of washings } & \multirow[b]{2}{*}{ Ethanol concentration } & \multicolumn{4}{|c|}{ Extract concentration ${ }^{*}$} \\
\hline & & $0 \%$ & $11.5 \%$ & $13.3 \%$ & $15.2 \%$ \\
\hline 1 & $63 \%$ & $\mathrm{X}$ & $\mathrm{X}$ & $\mathrm{X}$ & $\mathrm{X}$ \\
\hline 2 & $63 \%$ & $\mathrm{X}$ & $\mathrm{X}$ & $\mathrm{X}$ & $\mathrm{X}$ \\
\hline 1 & $45 \%$ & & & $\mathrm{X}$ & \\
\hline 1 & $40 \%$ & & & $\mathrm{X}$ & \\
\hline 1 & $35 \%$ & & & $\mathrm{X}$ & \\
\hline
\end{tabular}

${ }^{*}$ With respect to the initial volume.

TABLE 2: Characteristics of pectin obtained with different ratios extract to $99.8 \%$ ethanol.

\begin{tabular}{|c|c|c|c|c|c|}
\hline \multicolumn{6}{|c|}{ Ratio extract to $99.8 \%$ ethanol } \\
\hline \multicolumn{2}{|c|}{$1: 7$} & \multicolumn{2}{|c|}{$1: 4$} & \multicolumn{2}{|c|}{$1: 2$} \\
\hline$\% \mathrm{DM}$ & Acetylation degree & $\% \mathrm{DM}$ & Acetylation degree & $\% \mathrm{DM}$ & Acetylation degree \\
\hline $30.6 \pm 0.8$ & $1.6 \pm 0.1$ & $31.0 \pm 0.5$ & $1.5 \pm 0.1$ & $30.8 \pm 0.7$ & $1.9 \pm 0.1$ \\
\hline
\end{tabular}

Values are mean \pm standard deviations for $\mathrm{n}=3$.

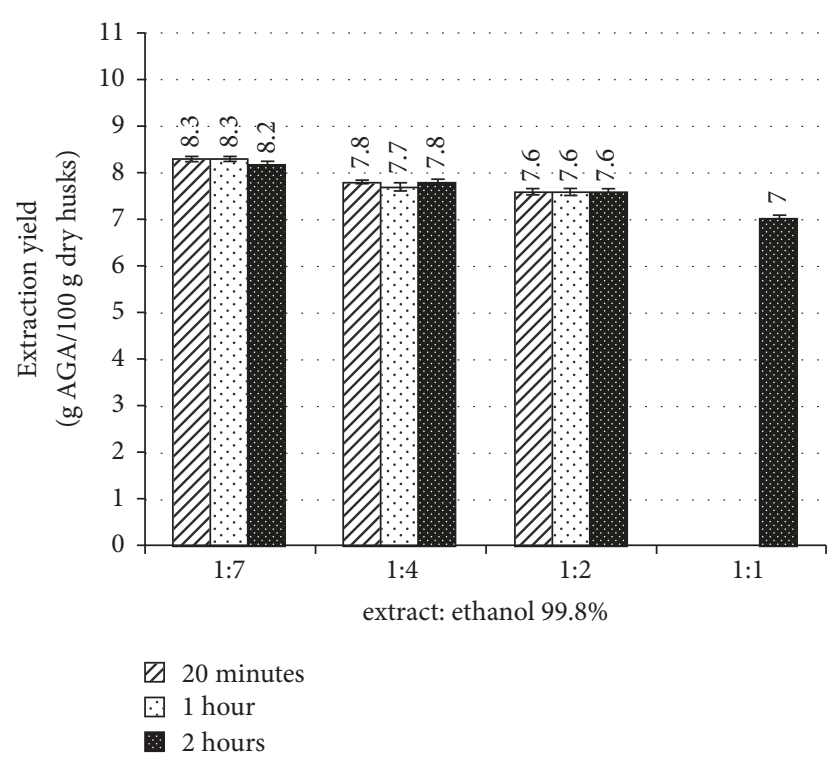

FIGURE 1: Pectin recovery (as g AGA/100 g dry husks) for the tested extract-to-ethanol ratios. Values are mean and the error bar shows standard deviations for $\mathrm{n}=3$.

decrement which becomes relevant if referred to the 1:7 run. Finally, for the lowest ethanol quantity (1:1), the recovered pectin reduces by more than a percentage point compared to the optimum precipitation conditions.

About the contact time, its prolongation did not give any benefit on the precipitated quantity for a given ratio extract to ethanol (runs with ratios 1:7, 1:4, and 1:2).

On the basis of these results it was decided to choose the ratio 1:4 and precipitation time of 20 minutes: these conditions are a good compromise considering extraction yield and saving of ethanol and processing time, on one side, and pectin loss, on the other. These operative conditions for

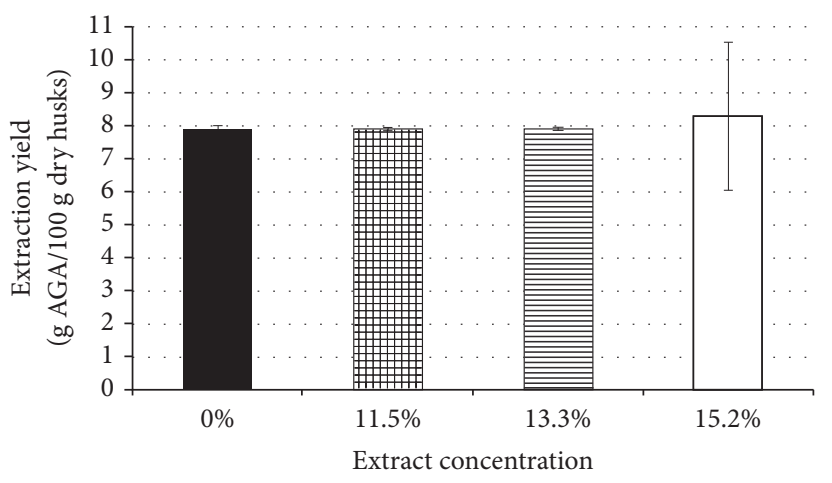

Figure 2: Pectin (as g AGA/100 g dry husks) obtained from extract concentrated at different value $(11.5,13.3$, and $15.5 \%)$. Results are mean and the error bar shows standard deviations for $\mathrm{n}=3$.

the precipitation step were kept as reference in the following runs.

As it is shown in Table 2, the simple reduction of the ethanol quantity does not cause alterations of pectin characteristics.

As for pectin obtained with the ratio 1:7, the product recovered with both ratios 1:4 and 1:2 is Low Methoxylated (LM) since its value is around 30 . The same conclusion is applicable to the acetylation degree which is always lower than $2 \%$.

3.2. Precipitation: Extract Concentration. Figure 2 shows that recovery yield is not influenced by the extract concentration up to $13.3 \%$. Vice versa, when the extract was concentrated by $15.2 \%$, the average yield resulted apparently higher, but this value is mean from a results set with high variability (over $27 \%$ as reported by error bars). The $15.2 \%$ concentrate had red color and was not completely transparent due to residual impurities still present after washing and interfering with 
TABLE 3: Influence of number of washings on pectin characteristics and extraction yield.

\begin{tabular}{|c|c|c|c|c|c|}
\hline & \multicolumn{5}{|c|}{ Extract concentration ${ }^{*}$} \\
\hline & $0 \%$ & $11.5 \%$ & $11.5 \%$ & $13.3 \%$ & $13.3 \%$ \\
\hline Number of washings & 1 & 1 & 2 & 1 & 2 \\
\hline Extraction yield (g AGA/100 g dry husks) & $8.2 \pm 0.1$ & $7.9 \pm 0.1$ & $8.0 \pm 0.1$ & $7.8 \pm 0.2$ & $8.1 \pm 0.1$ \\
\hline$\% \mathrm{DM}$ & $31.7 \pm 0.5$ & $31.5 \pm 0.6$ & $30.9 \pm 0.6$ & $31.0 \pm 0.5$ & $30.7 \pm 0.8$ \\
\hline Acetylation degree & $1.4 \pm 0.1$ & $1.6 \pm 0.1$ & $1.5 \pm 0.1$ & $1.9 \pm 0.1$ & $1.6 \pm 0.1$ \\
\hline
\end{tabular}

${ }^{*}$ With respect to the initial volume.

Values are mean \pm standard deviations for $n=3$.

TABLE 4: Characterization of pectin obtained with different ethanol concentrations in the washing operation.

\begin{tabular}{lccc}
\hline & \multicolumn{3}{c}{ Ethanol concentration } \\
& $40 \%$ & $45 \%$ & $63 \%$ \\
\hline Extraction yield (g & $8.1 \pm 0.1$ & $8.0 \pm 0.1$ & $7.8 \pm 0.2$ \\
AGA/100 g dry husks) & $32.0 \pm 0.6$ & $31.5 \pm 0.6$ & $31.0 \pm 0.5$ \\
$\% \mathrm{DM}$ & $1.8 \pm 0.1$ & $1.9 \pm 0.1$ & $1.9 \pm 0.1$ \\
\hline
\end{tabular}

Values are mean \pm standard deviations for $\mathrm{n}=3$.

the procedure utilized for the AGA determination. Other previous studies had already noticed the red color for the extractions with hydrochloric acid at $\mathrm{pH} 1.5$ and 1.0 [7] and with acetic acid at $\mathrm{pH} 1.6-2[10,11]$. Therefore, for all the following experiments the $13.3 \%$ concentrated extract was utilized, maintaining the ratio extract to ethanol equal to 1:4, while the rest of the procedure was kept unchanged.

3.3. Washing: Number of Operations. Considering that the initial extract is impure and impurities can interfere negatively with the final product, it was decided to modify the washing operation by doubling it (all the other operative conditions were not modified).

The results are reported in Table 3, and they show that the number of washings influences neither the quantity of recovered pectin nor the pectin quality. In addition, the test with $\alpha$-naphtol is negative for all the samples, confirming the absence of residual neutral sugars. At the same time, impurities such as polyphenols are absent.

These results demonstrate that two washings are not necessary and that a single operation with $63 \%$ ethanol is sufficient to obtain pectin with good purity level.

3.4. Washing: Reduction of Ethanol Concentration. For washing, three different concentrations of ethanol were tested, namely, 35,40 , and $45 \%$.

The $35 \%$ ethanol caused prolongation of the pectin separation.

Pectin obtained with 40 and $45 \%$ ethanol was analyzed in terms of quality and purity, and Table 4 reports the results, as g AGA/100 g dry husks, \%DM, and acetylation degree.

The data are very similar to those obtained with $63 \%$ ethanol, demonstrating that washing can be operated with $40 \%$ ethanol instead of higher concentrations.

\section{Conclusions}

The optimized operative conditions of the process for pectin recovery from roasted cocoa husks can be considered sustainable since good yields were achieved with reduced solvent quantity and working time.

In particular, the study demonstrated that it is possible to reduce ethanol for the precipitation using the ratio extract to ethanol equal to $1: 4$, while lower ratios give relevant pectin loss.

To reduce the ethanol quantity used for precipitation, the extract can be concentrated by $13.3 \%$ under vacuum at $60^{\circ} \mathrm{C}$ without changing pectin properties.

The precipitation seems to be not affected by time and, for this reason, the minimum duration equal to 20 minutes can be adopted.

At last, considering the purity of the final product, a single washing with $40 \%$ ethanol is sufficient.

Except for one condition, the experimental results showed good precision, demonstrated by the standard deviation values. Therefore, the optimized operative conditions can be considered rather reliable.

In the commonly used conditions the ethanol concentration is usually kept high also for washing (over 70\%) and with multiple operations (2-3 times). Therefore, comparing them to the ones presented in this paper, the changes showed a good saving of ethanol and processing time.

About its quality, the recovered pectin has low degree of methylation (around 31\%) and low degree of acetylation (< $2 \%$ ), therefore suitable for bakery jams and yogurt (if pectin final uses require higher $\% \mathrm{DM}$ values, treatments will have to be carried out).

As future perspective, these optimized data can be useful for a preliminary laboratory scale-up.

\section{Data Availability}

The data used to support the findings of this study are available from the corresponding author upon request.

\section{Conflicts of Interest}

The authors declare that they have no conflicts of interest.

\section{References}

[1] https://www.icco.org/home. 
[2] P. R. Ghosh, D. Fawcett, S. B. Sharma, and G. E. J. Poinern, "Progress towards sustainable utilisation and management of food wastes in the global economy," International Journal of Food Science, vol. 2016, Article ID 3563478, 22 pages, 2016.

[3] C. M. Galanakis, "Recovery of high added-value components from food wastes: conventional, emerging technologies and commercialized applications," Trends in Food Science \& Technology, vol. 26, no. 2, pp. 68-87, 2012.

[4] D. C. G. Okiyama, S. L. B. Navarro, and C. E. C. Rodrigues, "Cocoa shell and its compounds: applications in the food industry," Trends in Food Science \& Technology, vol. 63, pp. 103112, 2017.

[5] J. Serra Bonvehì and F. Ventura Coll, "Protein quality assessment in cocoa husk," Food Research International, vol. 32, no. 3, pp. 201-208, 1999.

[6] M. Plaza-Recobert, G. Trautwein, M. Pérez-Cadenas, and J. Alcañiz-Monge, "Preparation of binderless activated carbon monoliths from cocoa bean husk," Microporous and Mesoporous Materials, vol. 243, pp. 28-38, 2017.

[7] C. Mollea, F. Chiampo, and R. Conti, "Extraction and characterization of pectins from cocoa husks: a preliminary study," Food Chemistry, vol. 107, no. 3, pp. 1353-1356, 2008.

[8] S.-Y. Chan and W.-S. Choo, "Effect of extraction conditions on the yield and chemical properties of pectin from cocoa husks," Food Chemistry, vol. 141, no. 4, pp. 3752-3758, 2013.

[9] M. Masmoudi, S. Besbes, F. Abbes et al., "Pectin extraction from lemon by-product with acidified date juice: effect of extraction conditions on chemical composition of pectins," Food and Bioprocess Technology, vol. 5, no. 2, pp. 687-695, 2012.

[10] N. Ramli and Asmawati, "Effect of ammonium oxalate and acetic acid at several extraction time and $\mathrm{pH}$ on some physicochemical properties of pectin from cocoa husks (Theobroma cacao)," African Journal of Food Science, vol. 5, pp. 790-798, 2011.

[11] D. E. Marsiglia, K. A. Ojeda, M. C. Ramirez, and E. Sanchez, "Pectin extraction from cocoa pod husk (Theobroma cacao L.) by hydrolysis with citric and acetic acid," International Journal of ChemTech Research, vol. 9, no. 7, pp. 497-507, 2016.

[12] B. Min, J. Lim, S. Ko, K.-G. Lee, S. H. Lee, and S. Lee, "Environmentally friendly preparation of pectins from agricultural byproducts and their structural/rheological characterization," Bioresource Technology, vol. 102, no. 4, pp. 3855-3860, 2011.

[13] T. I. S. Oliveira, M. F. Rosa, F. L. Cavalcante et al., "Optimization of pectin extraction from banana peels with citric acid by using response surface methodology," Food Chemistry, vol. 198, pp. 113-118, 2016.

[14] J. R. Daniel, A. C. J. Voragen, and W. Pilnik, "Starch and other polysaccharides," in Ullman's Encyclopedia of Industrial Chemistry, F. T. Campbell and R. Pfefferkorn, Eds., vol. A25, pp. 24-34, VCH, 1994.

[15] N. Blumenkrantz and G. Asboe-Hansen, "New method for quantitative determination of uronic acids," Analytical Biochemistry, vol. 54, no. 2, pp. 484-489, 1973.

[16] T. J. Mangos and M. J. Haas, "Enzymatic determination of methanol with alcohol oxidase, peroxidase, and the chromogen 2,2l-azinobis(3-ethylbenzthiazoline-6-sulfonic acid) and its application to the determination of the methyl ester content of pectins," Journal of Agricultural and Food Chemistry, vol. 44, no. 10, pp. 2977-2981, 1996.

[17] D. R. Petrus and M. H. Dougherty, "Spectral characteristics of three varieties of florida orange juice," Journal of Food Science, vol. 38, no. 4, pp. 659-662, 1973. 


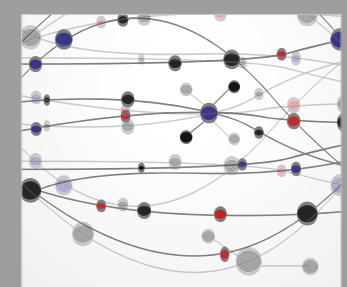

The Scientific World Journal
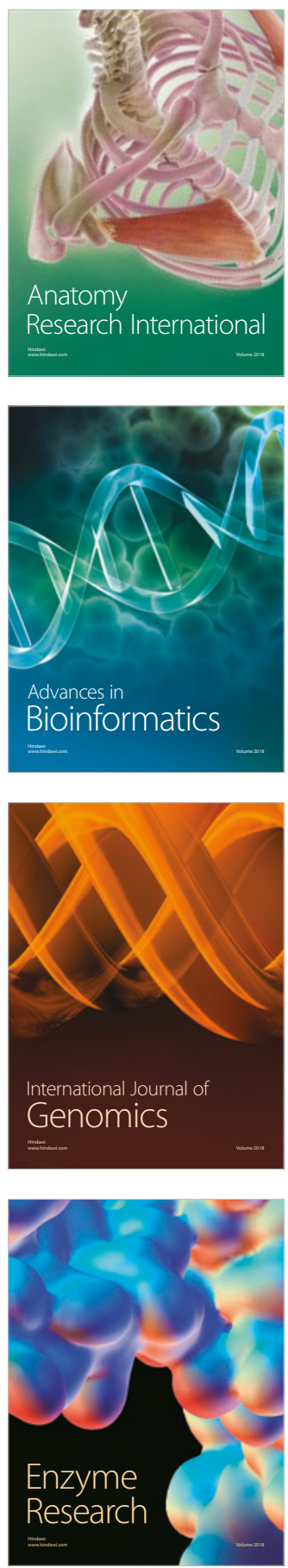
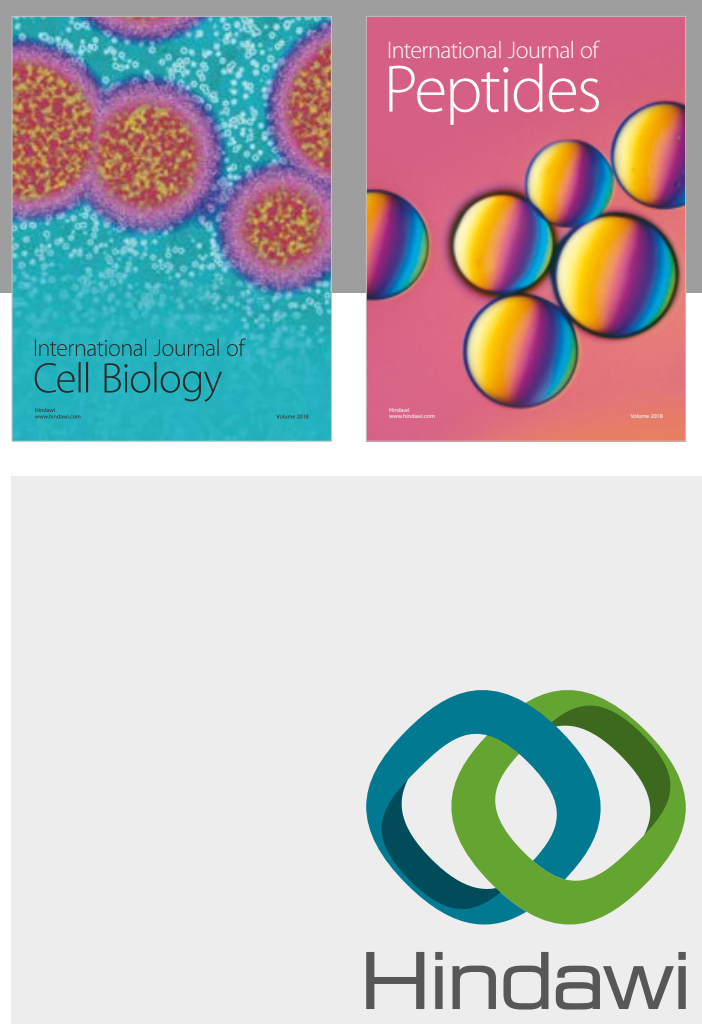

Submit your manuscripts at

www.hindawi.com
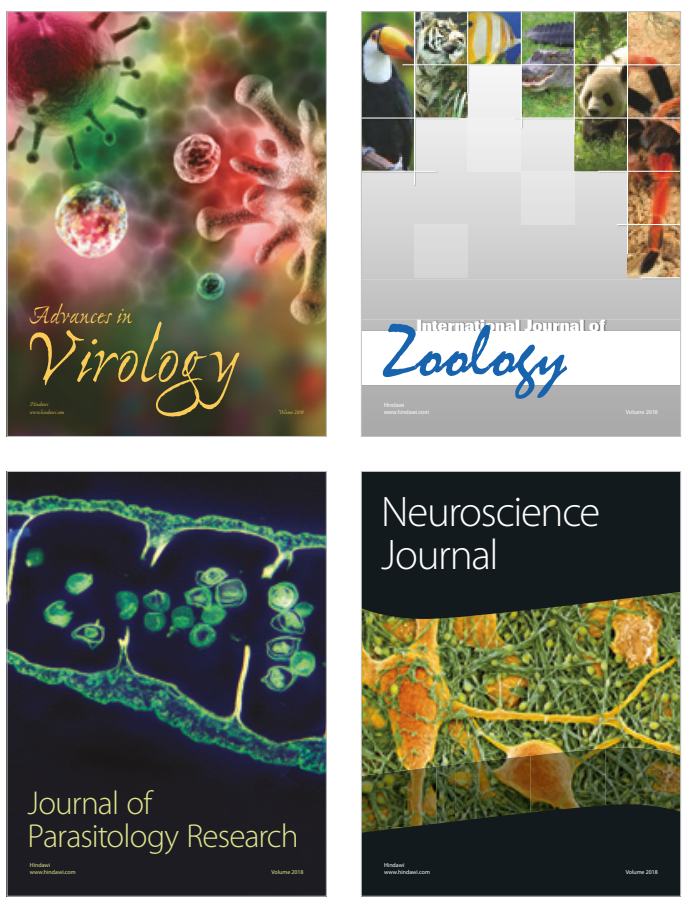
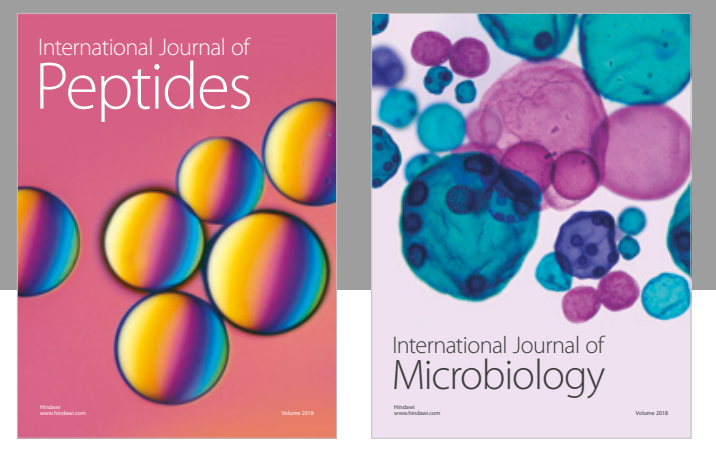

nternational Journal of Microbiology
Journal of
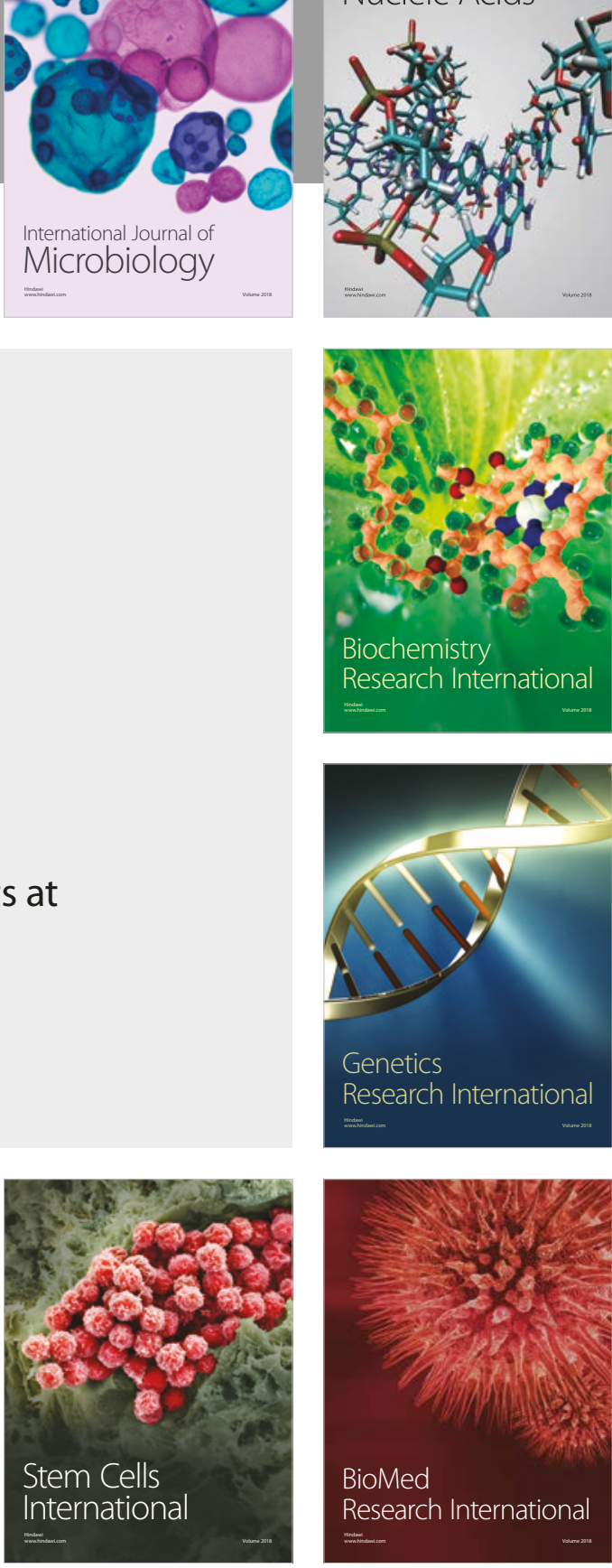
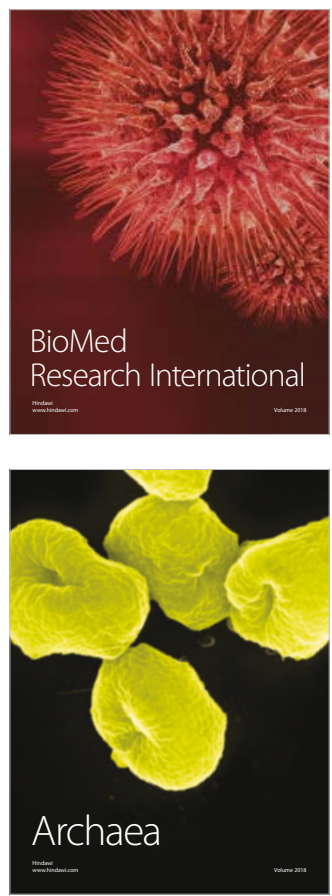